\title{
Research on Comprehensive Processing Technology of Gannan Navel Orange
}

\author{
Liming $\mathrm{Ou}^{1}$, Feipeng $\operatorname{Lin}^{2}$ \\ ${ }^{1}$ College of Food and Bioengineering, Jimei University \\ ${ }^{2}$ College of Information Engineering, Wuhan University of Technology
}

\begin{abstract}
Gannan navel orange has been well known since the 1970s. With the development of the country and society, navel orange has formed an overall system, including planting, production, storage, preservation, processing and other work processes. The technology of food processing with raw materials is becoming more and more extensive.
\end{abstract}

\section{Development of navel orange industry}

Gannan is an important city for the sale of fresh navel oranges and fruit export in China. Among the cities in China, Jiangxi, Hunan and Sichuan all have large-scale cultivation of navel oranges. Among them, Ganzhou City in Jiangxi is known as "the hometown of navel oranges in China." Jiangxi is located in a typical subtropical hilly mountainous area with a humid monsoon climate, coupled with abundant annual rainfall, sufficient sunlight, fertile soil and large temperature differences between day and night. The conditions for navel oranges to grow here make Jiangxi the largest production area of navel oranges in China. Gannan navel orange has been included in the national superior agricultural product regional plan for 2 consecutive years, and is called the product of national geographical indication product protection. Therefore, navel orange has also won the title of "Chinese famous fruit". In 2017, Gannan navel orange was included in the Central European "100+100" "Mutual recognition protection list.

Gannan navel orange fruit is large in shape, rich in nutrients, bright orange and red, smooth and beautiful, with an edible rate of $85 \%$. The meat is crisp and tender, and the residue is sweet and aromatic. It contains more than $55 \%$ juice. Because navel orange juice is rich, people love to eat fresh navel orange, and many food manufacturers carry out some processing on navel orange, so that it can not only ensure the nutritional value of navel orange itself but also can be used in a variety of forms, such as the processing of orange juice drinks, Research on processing of navel orange fruit vinegar, processing of orange-flavored jam, and processing technology of orange-flavored air freshener. It can be seen that navel orange can be used not only for food production, but also for the processing of daily necessities, so that the development scope of the navel orange industry has become broader.

\section{Study on processing technology of navel orange}

The processing technology can keep the nutritional value of navel orange itself, make the brewed wine unique in flavor, mellow in taste and rich in flavor. The development of fruit wine in China has only been more than ten years, and so far no mature and suitable sales model has been found, but the nutritional value of fruit wine itself has been slowly discovered by people. The era is developing rapidly. Now people generally pay attention to health, and the development of fruit wine industry has followed. Consumers' health needs not only occupy a certain market in China, but also the rapid development of fruit wine in foreign markets.

\subsection{Fruit wine effect}

"Fruit wine", as the name implies, is made from fruits or other foods with nutritional value, and most are made by fermentation of the fruit itself, without adding any other additives to ensure the nutritional value of the fruit wine, and the fruit wine made from fruit will also correspond to the fruit Image and nutritional value, for example, the appearance of apple cider is mostly yellow-green. Eating apple cider can accelerate the metabolism of the human body, promote blood circulation, relieve bowel movements and lose weight. When the summer climate is hot, people who are prone to heat stroke can also eat it. Similarly, navel orange is sour and cold, and has the function of appetizing and reducing vomiting. The fruit wine brewed from navel orange can treat stagnation and flatulence. Long-term use can also prevent chest pain, stomach pain, and food accumulation. It is suitable for the elderly and Women's consumption has a good health function.

Try to ensure that lines are no thinner than 0.25 point.

\footnotetext{
* Corresponding author: linfeipeng@,whut.edu.cn
} 


\subsection{Fruit wine brewing and processing}

Step 1: Pick fresh and ripe navel orange, wash it and dry the skin moisture, cut the head of navel orange with a knife, dig out the orange flesh, and keep the orange peel shell intact;

Step 2: Cut the minced orange flesh into small pieces, and put an appropriate amount into the processing machine for crushing to obtain crushed orange flesh, and then grind the crushed orange pieces into orange pulp with a refiner;

Step 3: Calculate the corresponding proportion of mixed enzyme and add it to the orange pulp and mix it evenly using a blender to obtain a uniformly mixed orange pulp, then pour the mixed orange pulp into the three-quarters of the orange peel shell, and then pour white wine (Liquor alcohol content is $40-60 \%$ ) until it is filled with orange peel shells, and finally sealed with the cut orange peel shells covered, and the outer cover of the oranges is sealed with a plastic wrap to obtain a fermentation device made by the oranges themselves;

(Note: The amount of mixed enzyme added is 0.02$0.08 \%$ of the mass of orange pulp. The mixed enzyme uses a mixed enzyme composed of cellulase and saccharification enzyme, and fruit wine yeast is added to the mixed enzyme)

Step 4: Place the finished fermentation device in the fermentation tank and arrange it in a neat manner, then put the bearing plate on the top of the fermentation tank, pour distilled water until the bearing plate is immersed, seal the fermentation tank, and ferment at a constant temperature of $35^{\circ} \mathrm{C}$ for $4-10$ days;

Step 5: Open the fermentation tank and pour out the distilled water, then blow hot air to dry the outer layer of the fermentation device, take out the fermentation device, and pour out the fermentation liquid in the orange peel shell;

Step 6: Put the fermentation broth into the separator 3000r for solid-liquid separation, the obtained supernatant is the fermentation broth; the aging process temperature is $5-10{ }^{\circ} \mathrm{C}$, time is $12-24 \mathrm{~h}$, to achieve a fruity rich, clear liquor In the state, the navel orange wine with pure aroma and high nutritional value is finally obtained.

\subsection{The nutritional value of jam}

Jam is a gel substance made by mixing fruits, sugar and acidity regulator, and cooking at a temperature over $100^{\circ} \mathrm{C}$, also called fruit paste. Making jam is a way to preserve fruits for a long time. It is mainly used on bread or toast. Regardless of the small fruits such as strawberries, blueberries, grapes, and rose fruits, or large fruits such as plums, oranges, apples, and peaches, they can also be made into jams. Only one fruit is usually used at the same time. Sugar-free jams, cheap jams or special jams (eg: durian, pineapple) will use colloids. Colloids commonly used in jams include: pectin, soy bean gum, and sage gum.

Jam is soft, sour and sweet, because it is made of various fruits and vegetables, so jam has its own extremely rich nutrients. The jam also contains the minerals and fibers needed by the human body, and some antioxidants contained in fruits such as flavonoids and anthocyanins. The pectin substance contained in the jam is still a very typical nutrient. It is actually a soft edible dietary fiber. Pectin is not only more absorbent than ordinary crude fiber (such as dietary fiber in Chinese cabbage), And there is no risk of harming the esophagus or gastrointestinal tract like crude fiber. Not only that, the activated pectin in the jam is also more capable of preventing cancer. In addition to pectin jam, it also contains natural fruit acid components, which can promote the secretion of digestive juice in the body, enhance the appetite of the person and help digestion. Edible jam can also increase the hemoglobin in the human body, and can well relieve the symptoms of iron deficiency anemia. There are also rich potassium and zinc elements in the jam that can reduce human fatigue and help increase memory. Many nutritional values of jam make its industry develop rapidly.

\subsection{Jam industry development}

According to the China Jam Industry Development Review and Development Trend Analysis Report (20182025) released by the China Industry Research Network, most of the domestic jam production companies are Chinese-foreign joint ventures. As of the relatively mature stage of the jam industry development, market competition is already in On a higher platform, the requirements for resources, $R \& D$, manufacturing and marketing have been greatly improved, and as the market concentration increases, the industry entry threshold will also be further increased. The existing processing capacity of domestic jams has become saturated. In order to ensure the long-term healthy development of the industry, production enterprises should not blindly expand in production scale. Instead, they should carry out resource integration and technology investment on the existing basis, and insist on quality innovation. Brand, expand the market with the brand.

\subsection{Making and processing of jam}

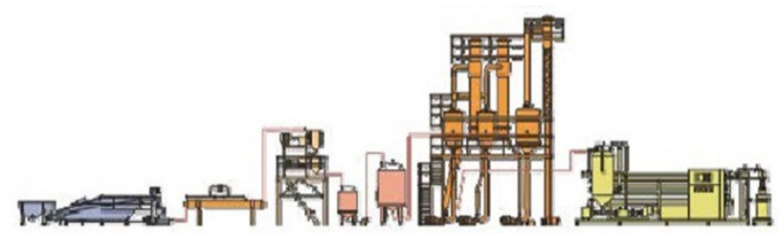

Fig. 1. Processing technology of jam

The raw material of the jam is navel orange peel, and its added substances are xylitol and citric acid, etc., and the $\mathrm{PH}$ value of the jam is 3-3.5.

Step 1: Pick fresh and ripe navel orange, peel off the orange peel and put it in an aqueous solution of sodium polyphosphate (mass percentage: $0.05 \%-0.15 \%$ ), soak 
for $1-3 \mathrm{~h}$, soaking temperature is $18-25^{\circ} \mathrm{C}$, And then taken out and washed, thereby obtaining the first softened orange peel;

Step 2: Place the first softened orange peel in the previous step in an aqueous $\mathrm{NaHCO} 3$ solution and cook for 2-4 minutes at a cooking temperature of $90-100^{\circ} \mathrm{C}$ to obtain a second softened orange peel;

Step 3: Place the second softened orange peel in an aqueous solution of $\mathrm{NaCl}$ (mass percentage: $0.3 \%-0.7 \%$ ), and scrub for 8-15 minutes to obtain an orange peel without bitterness;

Step 4: The debittered orange peel, xylitol, citric acid and water are mixed, placed in a beater for crushing for 5-10 minutes, and finally the orange peel pulp is concentrated to obtain jam.

Jam is suitable for people of any age. It also has health benefits for babies. Long-term use can prevent rickets in babies. The health function of jam makes its industrial development more rapid.

\section{Processing of essential oil extracted from orange peel}

In the current society where technology is developing rapidly, the use of various substances is becoming more and more full. The navel orange skin also has a place to use. The flesh of navel orange can be used for fresh food or wine, and the remaining navel orange skin can be used It is used to make jam for consumption, and it can also be used to extract a kind of aroma and extract essential oil for daily use. Navel orange peel has a sweet and sour aroma, people can relieve anxiety when using it, and it can also be used at night to help people get enough sleep.

\subsection{Process of extracting essential oil from orange peel}

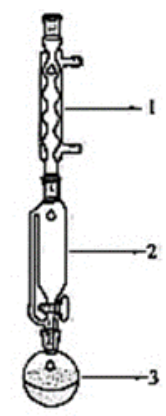

Fig. 2. Extraction of essential oil by distillation

Firstly, Taking a $25 \mathrm{ml}$ milled conical flask and sterilize it at a high temperature to facilitate the extraction of essential oils and reduce the generation of impurities. Then add $3 \mathrm{~g}$ of fresh orange peel to it and add distilled water until the orange peel has not passed. Connect the conical flask to a $25 \mathrm{ml}$ water separator, and use a hot plate heating device with adjustable temperature to gradually heat to a boiling state and maintain it for 4 minutes. After the solution is cooled, the extracted orange peel essential oil can be poured out from the upper port. The aroma of the obtained essential oil is pleasant and pleasant. This process is simple and easy to operate and is suitable for wide production.

\section{Conclusion}

Navel orange has high nutritional value. Both fresh food and processed products can retain their nutrients very well. The processing technology of navel orange can help the industry of navel orange develop rapidly. It also allows people to understand the correlation of navel orange through different types of products. Purpose, so it is very necessary to study the processing technology of navel orange, and we also need to devote ourselves to the development of research work.

\section{References}

1. Sun Sisheng, Guo Xiaohui, Li Guanghui. Study on the production process of apple jam. Journal of Xuchang University, 38(02): 84-88. (2019)

2. Cheng Shaonan. The characteristic aroma, nutrients and health care functions of oranges. China Fruit Industry Information, 31(12): 70-73. (2014)

3. Zeng Zhaoqiong. Organic Chemistry Experiment. Higher Education Press, (1987)

4. Gu Yiqiang. Discussion on influencing factors and safeguard measures of food safety. Light Industry Standards and Quality. (2020)

5. Jiang Kaixin, Ma Liqing. Application progress of sterilization technology in food processing. Food Safety Guide. (2020)

6. He Yingdong, Cui Wenjia, Li Xiao, Wang Yueming, Wang Wenliang, Tang Xiaozhen. The mechanism of action of pectin methylesterase and its application in food processing. Food Research and Development, 41(12): 185-189 . (2020)

7. Chen Shiqing, Wang Zhengzheng, Yao Siminwei, Luo Jing, Li Yufeng. Processing technology of kiwi low sugar compound jam. New Rural Technology, (2020)

8. Peng $\mathrm{Xu}$. On the planting technology and freshkeeping method of Gannan navel orange. Shanxi Agricultural Economics, (2019)

9. Guo Xiaohui, Sun Sisheng, Li Peirui, Zhang Houxiang. Preparation of orange jam. Journal of Xuchang University, 38(05): 101-105, (2019)

10. Shu Chang. The exploration of the path of modernization of Gannan navel orange industry. Northern Economy and Trade, (2019)

11. Liu Ke. The extraction of essential oil from Gannan navel orange and its antibacterial and anticancer activity. Gannan Normal University, (2019).

12. Sun Sisheng, Guo Xiaohui, Li Guanghui. Study on the production process of apple jam. Journal of Xuchang University, 38(02): 84-88, (2019) 
13. Cheng Shaonan. The characteristic aroma, nutrients and health care functions of oranges. China Fruit Industry Information, 31(12): 70-73. (2014) 